\title{
Gene Regulation During Cold Stress Acclimation in Plants
}

\author{
Viswanathan Chinnusamy, Jian-Kang Zhu, and Ramanjulu Sunkar
}

\begin{abstract}
Cold stress adversely affects plant growth and development and thus limits crop productivity. Diverse plant species tolerate cold stress to a varying degree, which depends on reprogramming gene expression to modify their physiology, metabolism, and growth. Cold signal in plants is transmitted to activate CBF-dependent (C-repeat/drought-responsive element binding factordependent) and CBF-independent transcriptional pathway, of which CBF-dependent pathway activates $\mathrm{CBF}$ regulon. $\mathrm{CBF}$ transcription factor genes are induced by the constitutively expressed ICE1 (inducer of CBF expression 1) by binding to the $C B F$ promoter. ICE1-CBF cold response pathway is conserved in diverse plant species. Transgenic analysis in different plant species revealed that cold tolerance can be significantly enhanced by genetic engineering CBF pathway. Posttranscriptional regulation at pre-mRNA processing and export from nucleus plays a role in cold acclimation. Small noncoding RNAs, namely micro-RNAs (miRNAs) and small interfering RNAs (siRNAs), are emerging as key players of posttranscriptional gene silencing. Cold stressregulated miRNAs have been identified in Arabidopsis and rice. In this chapter, recent advances on cold stress signaling and tolerance are highlighted.
\end{abstract}

\section{Keywords}

Cold stress; second messengers; CBF regulon; CBF-independent regulation; ICE1; posttranscriptional gene regulation

\section{Introduction}

Temperature profoundly influences the metabolism of organisms and thus is a key factor determining the growing season and geographical distribution of plants. Cold stress can be classified as chilling $\left(<20^{\circ} \mathrm{C}\right)$ and freezing $\left(<0^{\circ} \mathrm{C}\right)$ stress. Temperate plants have evolved a repertoire of adaptive mechanisms such as seed and bud dormancy, photoperiod sensitivity, vernalization, supercooling (prevention of ice formation in xylem parenchyma cells up to homogenous ice nucleation temperature, $-40^{\circ} \mathrm{C}$ ), and cold acclimation. In cold acclimation, plants acquire freezing tolerance on prior exposure to suboptimal, low, nonfreezing temperatures. The molecular basis of cold acclimation and acquired freezing tolerance in Arabidopsis and winter cereals has been studied extensively. Plants modify their metabolism and growth to adapt to cold stress by reprogramming gene expression during cold acclimation $(1,2)$. This chapter briefly covers cold stress signaling, transcriptional and posttranscriptional regulation of gene expression in cold acclimation process, and the genetic engineering of crops with enhanced cold tolerance.

\section{Cold Stress Sensing}

Thus far, the identity of stress sensor in plants is unknown. The fluid mosaic physical state of the plasma membrane is vital for the structure and function of cells, as well as to sense temperature stress. The plasma membrane undergoes phase transitions, from a liquid crystalline to a rigid gel phase at low temperature and to a fluid state at high temperature. Thus, a decrease in temperature can rapidly induce membrane rigidity at microdomains. 
Further, protein folding is influenced by temperature changes. Temperature-induced changes in the physical state of membranes and proteins are expected to change the metabolic reactions and thus the metabolite concentrations. Therefore, plant cells can sense cold stress through membrane rigidification, protein/nucleic acid conformation, and/or metabolite concentration (a specific metabolite or redox status).

In alfalfa and Brassica napus, cold stress-induced plasma membrane rigidification leads to actin cytoskeletal rearrangement, induction of $\mathrm{Ca}^{2+}$ channels, and increased cytosolic $\mathrm{Ca}^{2+}$ level. These events induce the expression of cold-responsive (COR) genes and cold acclimation. Further, a membrane rigidifier (DMSO) can induce $C O R$ genes even at $25^{\circ} \mathrm{C}$, whereas a membrane fluidizer (benzyl alcohol) prevents $C O R$ gene expression even at $0^{\circ} \mathrm{C}$ $(3,4)$. Genetic evidence for plants sensing cold stress through membrane rigidification is from the study of the fad 2 mutant impaired in the oleic acid desaturase gene of Arabidopsis. In wild-type Arabidopsis plants, diacylglycerol (DAG) kinase is induced at $14^{\circ} \mathrm{C}$. The $f a d 2$ mutant (more saturated membrane) and transgenic Arabidopsis overexpressing linoleate desaturase gene showed the expression of DAG kinase at 18 and $12^{\circ} \mathrm{C}$, respectively (5).

\section{Second Messengers and Signaling}

Cytosolic $\mathrm{Ca}^{2+}$ levels act as second messenger of the cold stress signal (6). Calcium may be imported into the cell or released from intracellular calcium stores. Patch-clamp studies of cold-induced potential changes of the plasma membrane in Arabidopsis mesophyll protoplasts showed the cold-activatedcalcium-permeable channel involved in the regulation of cytosolic $\mathrm{Ca}^{2+}$ signatures (7). Membrane rigidification induced cytosolic $\mathrm{Ca}^{2+}$ signatures; and $C O R$ gene expression was impaired by gadolinium, a mechanosensitive $\mathrm{Ca}^{2+}$ channel blocker, which suggests the involvement of mechanosensitive $\mathrm{Ca}^{2+}$ channels in cold acclimation (4). Pharmocological studies implicated cyclic ADP-riboseand inositol-1,4,5triphosphate $\left(\mathrm{IP}_{3}\right)$-activated intracellular calcium channels in COR gene expression (4). Calcium influx into the cell appears to activate phospholipase C (PLC) and D (PLD), which produce $\mathrm{IP}_{3}$ and phosphatidic acid, respectively. $\mathrm{IP}_{3}$ can further amplify $\mathrm{Ca}^{2+}$ signatures by activation of $\mathrm{IP}_{3}$-gated calcium channels (8). Genetic analysis revealed that loss-of-function mutants of FIERYI (FRYI) inositol polyphosphate 1-phosphatase show significantly higher and sustained levels of $\mathrm{IP}_{3}$ instead of the transient increase observed in wild-type plants. This situation leads to higher induction of COR genes and CBFs, the upstream transcription factors (9). In addition, the calcium exchanger 1 (caxl) mutant of Arabidopsis, which is defective in a vacuolar $\mathrm{Ca}^{2+} / \mathrm{H}^{+}$antiporter, exhibited enhanced expression of C-repeat binding factor/dehydration responsive element binding $(C B F / D R E B)$ proteins and their target $C O R$ genes (10). Therefore, cytosolic $\mathrm{Ca}^{2+}$ signatures are upstream of the expression of $C B F s$ and $C O R$ genes in cold stress signaling.

Cold acclimation induces accumulation of ROS such as $\mathrm{H}_{2} \mathrm{O}_{2}$, both in chilling-tolerant Arabidopsis and chilling-sensitive maize plants. ROS can act as a signaling molecule to reprogram transcriptome probably through induction of $\mathrm{Ca}^{2+}$ signatures and activation of mitogen-activated protein kinases (MAPKs) (11) and redox-responsive transcription factors. Arabidopsis frostbitel (frol) mutant, which is defective in the mitochondrial $\mathrm{Fe}-\mathrm{S}$ subunit of complex I (NADH dehydrogenase) of the electron transfer chain, shows a constitutively high accumulation of ROS. This high accumulation of ROS in frol results in reduced COR gene expression and hypersensitivity to freezing stress, probably because of desensitization of cells by the constitutively high ROS expression (12).

Cold stress-induced second messenger signatures can be decoded by different pathways. Calcium signatures are sensed by calcium sensor family proteins, namely calcium-dependent protein kinases (CDPKs), calmodulins (CaMs), and salt overly sensitive 3-like (SOS3-like) 
or calcineurin B-like (CBL) proteins. In a transient expression system in maize leaf protoplast, a constitutively active form of an Arabidopsis CDPK (AtCDPK1) activated the expression of barley HVAl ABA-responsive promoter::LUC reporter gene suggesting that AtCDPK is a positive regulator in stress-induced gene transcription (13). Genetic and transgenic analyses implicated CDPKs as positive regulators, but a calmodulin, a SOS3-like or a CBL calcium binding protein, and a protein phosphatase $2 \mathrm{C}$ (AtPP2CA) are negative regulators of gene expression and cold tolerance in plants. Components of MAPK cascades are induced or activated by cold and other abiotic stresses. Genetic and transgenic analyses showed that MAPKs act as a converging point in abiotic stress signaling. ROS accumulation under these stresses might be sensed through a MAPK cascade (14). ROS activates the AtMEKK1/ANP1 (MAPKKK)-AtMKK2 (MAPKK)-AtMPK4/6 (MAPK) MAPK cascade, which positively regulates cold acclimation in plants (11). Many of these phosphorylated proteins show activation or induction of gene expression under multiple stress conditions, and genetic modification results in alteration of multiple stress responses. These results suggest that the proteins act as connecting nodes of stress signal networks. Identification of the target proteins or transcription factors of protein kinase or phosphatase cascades will shed further light on stress signaling.

\section{Transcriptional Regulation}

Chilling-tolerant plants reprogram their transcriptome in response to acclimation temperature. Cold-regulated genes constitute about 4-20\% of the genome in Arabidopsis (15). The promoter region of many COR genes of Arabidopsis contains C-repeat (CRT)/ DREs, initially identified in the promoter of responsive to dehydration 29A (RD29A/COR78/ LTI78). As well, ABA-responsive elements are present in many cold-induced genes. Genetic screens using dehydration and cold stress-responsive promoter-driven LUCIFERASE (RD29A::LUC and CBF3::LUC) led to the isolation of mutants, which unraveled coldresponsive transcriptional networks.

\subsection{CBF Regulons and Cold Tolerance}

Yeast one-hybrid screens to identify CRT/DRE binding proteins led to the identification of CRT/DREBs (CBFs/DREBs) in Arabidopsis. CBFs belong to the ethylene-responsive element binding factor/APETALA2 (ERF/AP2)-type transcription factor family. Arabidopsis encodes three $C B F$ genes (CBF1/DREB1B, CBF2/DREB1C, and CBF3/ $D R E B 1 A$ ), which are induced within a short period of exposure to cold stress. CBFs bind to $C R T / D R E$ cis-elements in the promoters of COR genes and induce their expression $(16,17)$. Ectopic expression of $C B F s$ in transgenic Arabidopsis induced the expression of $C O R$ genes at warm temperatures and induced constitutive freezing tolerance. These transgenic Arabidopsis plants were also tolerant to salt and drought stresses (17-19). Microarray analysis of $C B F$-overexpressing transgenic plants identified several CBF target genes involved in signaling, transcription, osmolyte biosynthesis, ROS detoxification, membrane transport, hormone metabolism, and stress response (20,21). Transgenic overexpression of Arabidopsis CBFs is sufficient to induce cold tolerance in diverse plant species (Table 3.1). Further, CBF homologs have been identified from several chilling-tolerant and chillingsensitive plant species and transgenic analysis confirmed their pivotal role in cold acclimation (Table3.1).

These evidences suggest that a CBF transcription network plays a pivotal role in cold acclimation of evolutionarily diverse plant species. Transcriptome analysis of transgenic tomato and Arabidopsis plants overexpressing LeCBF1 and AtCBF3 revealed that CBF regulons from freezing-tolerant and freezing-sensitive plant species differ significantly (35). 
Constitutive overexpression of CBFs under the transcriptional control of the $35 \mathrm{~S}$ cauliflower mosaic virus promoter in transgenic plants resulted in severe growth retardation under normal growth conditions in diverse plant species such as Arabidopsis $(18,19,34,36), B$. napus (22), tomato (23,24), potato (29), and rice (31). Inhibition of metabolism and change in growth-regulating hormones appears to be important causes of the growth inhibition of CBF-overexpressing plants. Reduction in the expression of photosynthetic genes appears to reduce photosynthesis and growth under cold stress. Transgenic plants constitutively overexpressing CBFs showed higher induction of the STZ/ZAT10 zinc finger transcription factor gene, which appears to repress genes involved in photosynthesis and carbohydrate metabolism and thus reduce the growth of these transgenic plants (21). Microarray analysis revealed that cold stress regulates several genes involved in biosynthesis or signaling of hormones such as ABA, gibberellic acid (GA), and auxin, which suggests the importance of these hormones in coordinated regulation of cold tolerance and plant development (15). GA promotes important processes in plant growth and development, such as seed germination, growth through elongation, and floral transition. Growth retardation of transgenictomato plants constitutively overexpressing $A t C B F 1$ was reversed by $\mathrm{GA}_{3}$ treatment (24). This finding suggested a link between CBFs and GA in cold stress-induced growth retardation. During cold stress, growth retardation appears to be regulated by CBFs through nuclearlocalized DELLA proteins, which repress growth in Arabidopsis. GA stimulates the degradation of DELLA proteins and promotes growth. CBFs enhance the expression of GAinactivating GA2-oxidases, and thus allow the accumulation of the DELLA protein repressor of GA1-like 3 (RGL3), which leads to dwarfism and late flowering. Further, mutant plants of DELLA genes encoding GA-insensitive [GAI] repressor of GA1-3 [RGA] were significantly less freezing tolerant than were wild-type plants after cold acclimation. This finding suggests that DELLAs might contribute significantly to cold acclimation and freezing tolerance (37).

\subsection{Regulators of CBF Expression}

Transcription of CBF genes is induced by cold stress. Hence, constitutive transcription factors present in the cell at normal growth temperatures may induce the expression of CBFs on activation by cold stress. A systematic genetic analysis by $C B F 3:: L U C$ bioluminescent genetic screening led to the identification of a constitutively expressed and nuclear-localized transcription factor, inducer of CBF expression 1 (ICE1) in Arabidopsis. ICE1 encodes a MYC-type basic helix-loop-helix (bHLH) transcription factor, can bind to MYC recognition elements in the $C B F 3$ promoter, and induces the expression of $C B F 3$ during cold acclimation. The icel mutant is defective in both chilling and freezing tolerance, whereas transgenic Arabidopsis overexpressing ICEl showed enhanced freezing tolerance (38). Transcriptome analysis revealed the dominant icel mutant with impaired expression of about $40 \%$ of cold-regulated genes, in particular $46 \%$ of cold-regulated transcription factor genes (15). Therefore, ICE1 is a master regulator that controls CBF and many other coldresponsive regulons. Overexpression analysis showed that ICE2 (At1g12860, a homolog of ICE1) induces the expression of CBF1 and confers enhanced freezing tolerance in Arabidopsis after cold acclimation (39). In wheat, the ICE1 homologs TaICE141 and TaICE187 are constitutively expressed and activate the wheat CBF group IV, which are associated with freezing tolerance. Overexpression of TaICE141 and TaICE187 in Arabidopsis enhanced $C B F$ and CORgene expression and enhanced freezing tolerance only after cold acclimation. This finding suggests that similar to Arabidopsis ICE1, wheat ICE1 also needs to be activated by cold acclimation (40).

ICE1 appears to negatively regulate the expression of MYB15 (an R2R3-MYB family protein) in Arabidopsis. MYB15 is an upstream transcription factor that negatively regulates CBF expression. Transgenic Arabidopsis overexpressing MYB15 showed reduced 
expression of $C B F s$ and freezingtolerance, whereas myb15 T-DNA knockout mutants showed enhanced cold induction of $C B F s$ and enhanced freezing tolerance. In a yeast twohybrid system, ICE1 interacted with MYB15 (41). Further, the expression of MYB15 is increased in icel mutants (R236H and K393R) $(41,42)$. Thus, the ICE1-MYB15 interaction appears to play a role in regulating CBF expression levels during cold acclimation (41).

Although ICE1 is expressed constitutively, only on exposure to low temperature does it induce transcription of the CBF and other cold stress-responsive genes $(38,40)$.

Posttranslational modifications play a key role in regulating the activity of ICE1 under cold stress. Cold stress activates ICE1 sumoylation (42) and negatively regulates ICE1 levels by targeted proteolysis (43). The Arabidopsis High expression of Osmotically responsive gene 1 (HOSI) encodes a RING finger ubiquitin E3 ligase. The nuclear localization of $\mathrm{HOS} 1$ is enhanced by cold stress. HOS1 physically interacts with ICE1 and targets ICE1 for polyubiquitination and proteolysis of ICE1 after $12 \mathrm{~h}$ of cold stress. Overexpression of HOS1 in transgenic Arabidopsis results in a substantial reduction in level of ICE1 protein and that of its target genes, as well as hypersensitivity to freezing stress. Thus, HOS1 mediates ubiquitination of ICE1 and plays a critical role in maintaining the level of ICE1 target genes in the cell during cold acclimation (43). Sumoylation of proteins prevents the proteasomal degradation of target proteins. The null mutant of Arabidopsis SUMO E3 ligase, SAZ1 (SAP and Miz1), exhibits reduced cold induction of $C B F s$ and the target COR genes, as well as hypersensitivity to chilling and freezing stresses. SIZ1 catalyzes SUMO conjugation to K393 of ICE1 during cold acclimation and thus reduces polyubiquiti-nation of ICE1. Mutation in a K393 residue of ICE1 impairs its activity (42). Hence, SIZ1mediated sumoylation facilitates ICE1 stability and activity, whereas HOS1 mediation reduces ICE1 protein levels during cold acclimation.

Stomata play a crucial role in regulating photosynthesis and transpiration. Recently, the scream-D dominant mutant and icel mutant were found to be the same as $\mathrm{R} 236 \mathrm{H}$, which results in constitutive stomatal differentiation in the epidermis, and the entire epidermis differentiates into stomata. Thus, ICE1 is required for controlled stomatal development. ICE1protein interacts and forms a dimer with other bHLH transcription factors, SPEECHLESS (SPCH), MUTE, and FAMA, which regulate stomatal development. ICE1 may act as a link between the formation of stomata and the plant response to environmental cues (44).

Recently, members of the calmodulin binding transcription activator (CAMTA) family proteins have been identified as transcriptional regulators of $C B F 2$ expression. Cold-induced expression of $\mathrm{CBF} 2$ was considerably lower in camta3 mutant as compared to WT plants. The CAMTA3 protein binds to conserved DNA motifs present in $C B F 2$ promoter and regulates $C B F 2$ expression. The camtal/camta3 double mutant exhibited hyper-sensitivity to freezing stress as compared to WT plants. Since CAMTA proteins can interact with calmodulins, cold-induced calcium signals may regulate $C B F$ s expression through CAMTA proteins (45).

\subsection{CBF1, CBF2, and CBF3 Play Different Roles in Cold Acclimation}

Microarray analysis revealed that CBFs regulate about $12 \%$ of the cold-responsive transcriptome. Overexpression of CBFs enhances osmolyte accumulation, reduces growth, and enhances abiotic stress tolerance (Table 3.1). Constitutive overexpression studies of transgenic Arabidopsis suggested that $\mathrm{CBF} 1, \mathrm{CBF} 2$, and $\mathrm{CBF} 3$ have redundant functional activities (36). However, the ice 1 mutant, impaired mainly in $C B F 3$ but not $C B F 1$ and $C B F 2$, showed chilling and freezing hypersensitivity (38). Studies of the $c b f 2$ T-DNA insertion mutant of Arabidopsis revealed that CBFs have different functions in cold acclimation. cbf 2 null mutants showed increased expression of $C B F 1$ and $C B F 3$ and 
enhanced tolerance to freezing (with or without cold acclimation), dehydration, and salt stresses. Further, $C B F s$ show a temporal difference in expression, with the cold-induced expression of $C B F 1$ and $C B F 3$ preceding that of $C B F 2$. These results suggest that $\mathrm{CBF} 2$ negatively regulates $C B F 1$ and $C B F 3$ to optimize the expression of downstream target genes (45). In potato (Solanum tuberosum), overexpression of $A t C B F 2$ failed to confer freezing tolerance (29). Transgenic analysis of $C B F 1$ and $C B F 3$ RNAi lines revealed that both CBF1 and $\mathrm{CBF} 3$ are required for the full set of $\mathrm{CBF}$ regulon expression and freezing tolerance (46).

Besides $\mathrm{CBF} 2$, the $\mathrm{C} 2 \mathrm{H} 2$ zinc finger transcription factor ZAT12 negatively regulates the expression of $C B F 1, C B F 2$, and $C B F 3$ during cold stress. Arabidopsis transgenic plants overex-pressing ZAT12 showed decreased expression of $C B F s$ under cold stress (47). los 2 mutant plants showed an enhanced and more sustained induction of ZAT10/STZ during cold stress and enhanced cold sensitivity. LOS2 encodes a bifunctional enolase that negatively regulates the expression of ZAT10 (48).

Transgenic Arabidopsis plants overexpressing AtMKK2 showed constitutive expression of $C B F 2$, which suggests that the $C B F 2$ expression is probably positively regulated by a MAPK signaling cascade (11). Arabidopsis FIERY2 (FRY2), which encodes an RNApolymerase II C-terminal domain (CTD) phosphatase, appears to act as a negative regulator of $C B F s$ and their target $C O R$ genes because the fry 2 mutant showed enhanced expression of $C B F s$ and $C O R$ genesunder cold stress and ABA. Since the fry 2 mutant is hypersensitive to freezing despite enhanced expression of $C B F s$, FRY2 may positively regulate the expression of certain genes critical for freezing tolerance (49). The maintenance of an optimal level of CBFs at an appropriate time is necessary, because constitutive overexpression affects growth and development significantly. Further, $C B F$ expression is under the control of a circadian clock. The maximal cold-induced increase in transcription of CBFs occurs when cold stress is imposed $4 \mathrm{~h}$ after dawn. Transgenic plants overexpressing arrhythmic CCA1 showed no temporal difference in the cold induction of $C B F$ expression (50).

\subsection{CBF-Independent Regulons}

Genetic and transgenic analyses revealed that several classes of transcription factors besides CBFs play an important role in cold acclimation. The eskimol (eskl) mutant of Arabidopsis was identified through freezing tolerance genetic screening. The eskl mutant accumulated constitutively high levels of proline and exhibited constitutively freezing tolerance. ESKl is constitutively expressed and encodes the protein domain of unknown function (23). Transcriptome comparison of $\mathrm{CBF} 2$-overexpressing plants and esk1 mutants showed that different sets of genes are regulated by CBF2 and ESK1. However, the mechanism of action of ESK1 in freezing tolerance has yet to be revealed (51).

$P_{R D 29 A}: L U C$ reporter gene-based genetic screening led to the identification of two constitutively expressed transcription factors, HOS9 (a homeodomain protein) and HOS10 (an R2R3-type MYB), which are necessary for cold tolerance in Arabidopsis. hos 9 and hos 10 mutants are less freezing tolerant than wild-type Arabidopsis (52,53). Transcriptome analysis revealed distinct CBF and HOS9 regulons (52). HOS10 probably regulates ABAdependent cold acclimation pathways, because HOS10 positively regulates NCED3 (9-cisepoxycarotenoid dioxygenase) and thus ABA accumulation during cold stress (53).

Gene expression analysis revealed several transcription factors induced during cold acclimation. Transgenic analysis of cold-inducible transcription factors helped in validation of functions of some transcription factors in cold tolerance. Constitutive overexpression of the soybean $\mathrm{C} 2 \mathrm{H} 2$-type zinc finger protein SCOF1 in Arabidopsis transgenic plants 
enhanced the expression of $C O R$ genes and conferred constitutive freezing tolerance.

SCOF1 interacts with soybean G-box binding factor 1 (SGBF1) and may enhance the DNA binding activity of the SGBF1. SGBFl is induced by both cold and ABA (54).

Overexpression of the cold-regulated rice transcription factors MYB4 (an R2R3-type MYB) and $O S M Y B 3 R-2$ (an R1R2R3 MYB) enhanced freezing tolerance of Arabidopsis $(55,56)$.

Some members of the abiotic, plant hormone, and pathogen-inducible ERF family play a crucial role in abiotic and biotic stress tolerance. The pepper ERF/AP2-type transcription factor Capsicum annuum pathogen and freezing tolerance-related protein $1(\mathrm{CaPF1})$ is induced by cold, osmotic stress, ethylene, and jasmonic acid. Transgenic Arabidopsis overexpressing $C a P F 1$ showed induction of pathogen-responsive as well as $C O R$ genes and exhibited enhanced tolerance to stress by freezing and to pathogens (Pseudomonas syringae pv tomato DC3000) (57). Similarly, Triticum aestivum ERF1 (TaERF1] was induced by cold, drought salinity, ABA, ethylene, salicylic acid, and infection by Blumeria graminis $f$. sp. Tritici pathogen in wheat. Transgenic Arabidopsis overexpressing TaERF1 exhibited enhanced tolerance to cold, salt, and drought stresses, as well as pathogens (58). Genes encoding the A-5 subgroup AP2 domain protein from Physcomitrella patens (PpDBF1) (59) and soybean (GmDREB3) (60) are cold induced, and overexpression of these genes conferred enhanced cold tolerance.

In wheat, wheat low-temperature-induced protein 19 (WLIP19), encoding a basic-region leucine zipper protein, is induced by cold, drought, and ABA. WLIP19 activates the expression of COR genes in wheat. Transgenic tobacco overexpressing Wlip19 showed significant freezing tolerance. WLIP19 was found to interact and form a heterodimer with T.aestivum ocs-element bindingfactor 1 (TaOBF1), a bZIP transcription factor (61). The plant-specific transcription factor NAC (NAM, ATAF, and CUC) family plays a key role in stress response. Overexpression of cold stress-inducible rice $S N A C 2$ in transgenic rice resulted in high cell membrane stability under cold stress. Microarray analysis showed upregulation of several stress-regulated genes in SNAC2-overexpressing plants (62). These results suggest that several transcriptional networks operate during cold acclimation and cold stress tolerance of plants.

\section{Posttranscriptional Gene Regulation}

Posttranscriptional regulation at pre-mRNA processing, mRNA stability, and export from nucleus plays critical roles in cold acclimation and cold tolerance (2).

\subsection{Messenger RNA Processing}

Pre-mRNA processing and exports constitute important mechanisms of regulation of gene expression in eukaryotes. Pre-mRNA undergoes various nuclear processes such as the addition of a $5^{\prime}$ methyl cap and poly(A) tail and intron splicing. Splicing is necessary to remove introns and to synthesize translationally competent mRNAs. Primary transcripts with more than one intron can undergo alternative splicing to produce functionally different proteins from a single gene. In plants, about $20 \%$ of genes undergo alternative splicing. Although most alternative splicing events are uncharacterized in plants, but it appears to play an important role in the regulation of photosynthesis, flowering, grain quality in cereals, and plant defense response. Recent studies have implicated intron splicing in abiotic stress response. In wheat, cold stress induction of two early cold-regulated (e-cor) genes coding for a ribokinase (7H8) and a $\mathrm{C} 3 \mathrm{H} 2 \mathrm{C} 3 \mathrm{RING}$ finger protein (6G2) undergo stressdependent splicing. Both of these genes are regulated by intron retention under cold stress, whereas $6 \mathrm{G} 2$ intron retention is also regulated by drought stress. However, homologs of these genes did not show stress-regulated intron retention in Arabidopsis. Interestingly, barley homologs of 7H8 and 6G2 showed stress-dependent intron retention under cold 
stress, whereas barley albino mutants defective in chloroplast development failed to retain introns in these genes under cold stress (63). The Arabidopsis COR15A gene encoding a chloroplast stromal protein with cryoprotective activity plays an important role in conferring freezing tolerance to chloroplasts (64). The Arabidopsis stabilizedl (stal) mutant is defective in the splicing of the cold-induced COR15A pre-mRNA and is hyper-sensitive to chilling, ABA, and salt stresses. STAl encodes a nuclear pre-mRNA splicing factor and is upregulated by cold stress. STA1 catalyzes splicing of COR15A, which is necessary for cold tolerance (65). Further, pre-mRNA of serine/arginine-rich (SR) proteins, which are involved in the regulation or execution of mRNA splicing, undergo alternate splicing under cold and heat stresses in Arabidopsis (66). Further, in addition to a change in splicing pattern, expression levels of AtSR45a and AtSR30, SF2/ASF-like SR proteins, are also increased by high light and salinity stresses in Arabidopsis (67). Thus, the stress-regulated alternate splicing machinery may in turn change the splicing pattern of some of the stress-responsive genes.

\subsection{Small RNAs}

Small noncoding RNAs, namely micro-RNAs (miRNAs) and small interfering RNAs (siRNAs), act as ubiquitous repressors of gene expression in animals and plants. Small RNAs are incorporated into the argonaute (AGO) family of proteins containing the RNAinduced silencing complex (RISC) or RNA-induced transcriptional silencing (RITS) complex. The RISC-containing miRNA/siRNA induces posttranscriptional gene silencing by cleavage of mRNA and translational repression. Transcriptional gene silencing is mainly mediated by siRNAs. Cold stress-upregulated and -downregulated miRNAs have been identified in Arabidopsis. Abiotic stress-induced or -upregulated small RNAs can downregulate their target genes, which are likely negative regulators and/or determinants of the stress response. In contrast, stress-downregulated small RNAs can upregulate their target mRNAs, which are likely positive regulators and/or determinants of stress tolerance (68).

Accumulation of ROS is induced by abiotic stresses. Super-oxide dismutases catalyze conversion of the superoxide radical into $\mathrm{H}_{2} \mathrm{O}_{2}$, which is then detoxified by ascorbate peroxidase. The miR398 expression is reduced and that of its target genes CSD1 and CSD2 enhanced under oxidative stress in Arabidopsis. Under normal conditions, miR398 targets the CSD mRNAs for cleavage, and thus stress-induced reduction in miR398 expression results in accumulation of CSD transcripts. Because miR398 and its target sequence on the CSD mRNAs are conserved across plant species, miR398 appears to play a ubiquitous role in ROS detoxification under abiotic stresses (69). siRNAs derived from double-stranded RNAs (dsRNAs) formed from the mRNAs encoded by a natural cis-antisense gene pair are called natural antisense transcript-derived siRNAs (nat-siRNAs). One of the nat-siRNAs derived from a cis-nat pair of SRO5 and P5CDH ( $\triangle 1$-pyrroline-5-carboxylate dehydrogenase) regulates oxidative stress and osmolyte accumulation under salt stress in Arabidopsis. Salt stress-induced expression of SRO5 leads to SRO5-P5CDH dsRNA, which is then processed by DCL2, RDR6, SGS3, and DNA-dependent RNA polymerase IV (NRPD1A) to generate a 24-nt nat-siRNA. The 24-nt nat-siRNA targets the cleavage of $\mathrm{P} 5 \mathrm{CDH}$ and thus accumulation of proline. Oxidative stress also induces the expression of SRO5 and the 24-nt SRO5-P5CDH nat-siRNA and decreases P5CDH transcript levels $(70,71)$. These results suggest that small RNAs play a key role in gene regulation in the cold and other abiotic stress response of Arabidopsis.

\section{Conclusions and Perspectives}

Significant progress has been made to unravel the molecular basis of cold acclimation in model plant Arabidopsis and winter cereals. During cold acclimation, plants reprogram their gene expression through transcriptional, posttranscriptional, and posttranslational 
mechanisms. The ICE1-CBF transcriptional cascade plays crucial role in cold acclimation in diverse plant species. Transgenic analysis revealed that genetic engineering of CBF pathway can improve cold tolerance across plant species. Recently, several components of CBFindependent transcriptional pathway of cold acclimation have been identified. Besides transcriptional regulation, plants employ diverse posttranscriptional regulatory mechanisms to regulate their gene expression during cold acclimation. Several cold stress-regulated miRNAs have been identified in Arabidopsis and rice. Characterization of cold-regulated miRNAs will help understand the role of posttranscriptional regulation of mRNA stability in cold stress response of plants. Cold-induced transcriptome differs significantly among leaf, root, and reproductive (pollen) tissues. Most of the mechanisms of cold acclimation were studied in vegetative stages of Arabidopsis. Further studies on transcriptional networks in reproductive tissues will identify key regulators of cold tolerance. Epigenetic processes play a key role in the regulation of plant development and stress responses. Further studies on the function of epigenetic processes, such as DNA methylation and chromatin modifications, and epigenetic stress memory will be necessary.

\section{References}

1. Yamaguchi-Shinozaki K, Shinozaki K. Transcriptional regulatory networks in cellular responses and tolerance to dehydration and cold stresses. Annu Rev Plant Biol. 2006; 57:781-803. [PubMed: 16669782]

2. Chinnusamy V, Zhu J, Zhu JK. Cold stress regulation of gene expression plants. Trends Plant Sci. 2007; 12:444-451. [PubMed: 17855156]

3. Orvar BL, Sangwan V, Omann F, Dhindsa R. Early steps in cold sensing by plant cells: the role of actin cytoskeleton and membrane fluidity. Plant J. 2000; 23:785-794. [PubMed: 10998189]

4. Sangwan V, Foulds I, Singh J, Dhindsa RJ. Cold activation of Brassica napus BN115 promoter is mediated by structural changes in membranes and cytoskeleton, and requires $\mathrm{Ca}^{2+}$ influx. Plant $\mathrm{J}$. 2001; 27:1-12. [PubMed: 11489178]

5. Vaultier MN, Cantrel C, Vergnolle C, Justin A-M, Demandre C, Benhassaine-Kesri G, Cicek D, Zachowski A, Ruelland E. Desaturase mutants reveal that membrane rigidification acts as a cold perception mechanism upstream of the diacylglycerol kinase pathway in Arabidopsis cells. FEBS Lett. 2006; 580:4218-4223. [PubMed: 16839551]

6. Knight MR. Signal transduction leading to low-temperature tolerance in Arabidopsis thaliana. Philos Trans R Soc Lond B Biol Sci. 2002; 357:871-875. [PubMed: 12171650]

7. Carpaneto A, Ivashikina N, Levchenko V, Krol E, Jeworutzki E, Zhu JK, Hedrich R. Cold transiently activates calcium-permeable channels in Arabidopsis mesophyll cells. Plant Physiol. 2007; 143:487-494. [PubMed: 17114272]

8. Vergnolle C, Vaultier MN, Taconnat L, Renou JP, Kader JC, Zachowski A, Ruell E. The coldinduced early activation of phospholipase $\mathrm{C}$ and $\mathrm{D}$ pathways determines the response of two distinct clusters of genes in Arabidopsis cell suspensions. Plant Physiol. 2005; 139:1217-1233. [PubMed: 16258011]

9. Xiong L, Lee BH, Ishitani M, Lee H, Zhang C, Zhu JK. FIERY1 encoding an inositol polyphosphate 1-phosphatase is a negative regulator of abscisic acid and stress signaling in Arabidopsis. Genes Dev. 2001; 15:1971-1984. [PubMed: 11485991]

10. Catala R, Santos E, Alonso JM, Ecker JR, Martinez-Zapater JM, Salinas J. Mutations in the $\mathrm{Ca}^{2+}$ / $\mathrm{H}^{+}$transporter CAX1 increase CBF/DREB1 expression and the cold-acclimation response in Arabidopsis. Plant Cell. 2003; 15:2940-2951. [PubMed: 14630965]

11. Teige M, Scheikl E, Eulgem T, Doczi R, Ichimura K, Shinozaki K, Dangl JL, Hirt H. The MKK2 pathway mediates cold and salt stress signaling in Arabidopsis. Mol Cell. 2004; 15:141-152. [PubMed: 15225555]

12. Lee BH, Lee H, Xiong L, Zhu JK. A mitochondrial complex I defect impairs cold-regulated nuclear gene expression. Plant Cell. 2002; 14:1235-1251. [PubMed: 12084824]

13. Sheen J. Specific $\mathrm{Ca}^{2+}$-dependent protein kinase in stress signal transduction. Science. 1996; 274:1900-1902. [PubMed: 8943201] 
14. Pitzschke A, Hirt H. Mitogen-activated protein kinases and reactive oxygen species signaling in plants. Plant Physiol. 2006; 141:351-356. [PubMed: 16760487]

15. Lee BH, Henderson DA, Zhu JK. The Arabidopsis cold-responsive transcriptome and its regulation by ICE1. Plant Cell. 2005; 17:3155-3175. [PubMed: 16214899]

16. Stockinger EJ, Gilmour SJ, Thomashow MF. Arabidopsis thaliana CBF1 encodes an AP2 domaincontaining transcription activator that binds to the $\mathrm{C}$ repeat/DRE, a cis-acting DNA regulatory element that stimulates transcription in response to low temperature and water deficit. Proc Natl Acad Sci USA. 1997; 94:1035-1040. [PubMed: 9023378]

17. Liu Q, Kasuga M, Sakuma Y, Abe H, Miura S, Yamaguchi-Shinozaki K, Shinozaki K. Two transcription factors, DREB1 and DREB2, with an EREBP/AP2 DNA-binding domain separate two cellular signal transduction pathways in drought- and low-temperature-responsive gene expression in Arabidopsis. Plant Cell. 1998; 10:1391-1406. [PubMed: 9707537]

18. Jaglo-Ottosen KR, Gilmour SJ, Zarka DG, Schabenberger O, Thomashow MF. Arabidopsis CBF1 overexpression induces COR genes and enhances freezing tolerance. Science. 1998; 280:104-106. [PubMed: 9525853]

19. Kasuga M, Liu Q, Miura S, Yamaguchi-Shinozaki K, Shinozaki K. Improving plant drought, salt, and freezing tolerance by gene transfer of a single stress inducible transcription factor. Nat Biotech. 1999; 17:287-291.

20. Fowler S, Thomashow MF. Arabidopsis transcriptome profiling indicates that multiple regulatory pathways are activated during cold acclimation in addition to the CBF cold response pathway. Plant Cell. 2002; 14:1675-1690. [PubMed: 12172015]

21. Maruyama K, Sakuma Y, Kasuga M, Ito Y, Seki M, Goda H, Shimada Y, Yoshida S, Shinozaki K, Yamaguchi-Shinozaki K. Identification of cold-inducible downstream genes of the Arabidopsis DREB1A/CBF3 transcriptional factor using two microarray systems. Plant J. 2004; 38:982-993. [PubMed: 15165189]

22. Jaglo KR, Kleff S, Amundsen KL, Zhang X, Haake V, Zhang JZ, Deits T, Thomashow MF. Components of the Arabidopsis C-repeat/dehydration responsive element binding factor coldresponse pathway are conserved in Brassica napusand other plant species. Plant Physiol. 2001; 127:910-917. [PubMed: 11706173]

23. Hsieh TH, Lee JT, Charng YY, Chan MT. Tomato plants ectopically expressing Arabidopsis CBF1 show enhanced resistance to water deficit stress. Plant Physiol. 2002; 130:618-26. [PubMed: 12376629]

24. Hsieh TH, Lee JT, Yang PT, Chiu LH, Charng YY, Wang YC, Chan MT. Heterology expression of the Arabidopsis C-repeat/dehydration response element binding factor 1 gene confers elevated tolerance to chilling and oxidative stresses in transgenic tomato. Plant Physiol. 2002; 129:10861094. [PubMed: 12114563]

25. Kasuga M, Miura S, Shinozaki K, Yamaguchi-Shinozaki K. A combination of the Arabidopsis $D R E B 1 A$ gene and stress-inducible RD29A promoter improved drought- and low-temperature stress tolerance in tobacco by gene transfer. Plant Cell Physiol. 2004; 45:346-350. [PubMed: 15047884]

26. Pellegrineschi A, Reynolds M, Pacheco M, Brito RM, Almeraya R, Yamaguchi-Shinozaki K, Hoisington D. Stress-induced expression in wheat of the Arabidopsis thaliana DREB1A gene delays water stress symptoms under greenhouse conditions. Genome. 2004; 47:493-500. [PubMed: 15190366]

27. Oh SJ, Song SI, Kim YS, Jang HJ, Kim SY, Kim M, Kim YK, Nahm BH, Kim JK. Arabidopsis $C B F 3 / D R E B 1 A$ and $A B F 3$ in transgenic rice increased tolerance to abiotic stress without stunting growth. Plant Physiol. 2005; 138:341-351. [PubMed: 15834008]

28. Al-Abed D, Madasamy P, Talla R, Goldman S, Rudrabhatla S. Genetic engineering of maize with the Arabidopsis DREB1A/CBF3 gene using split-seed explants. Crop Sci. 2007; 47:2390-2402.

29. Pino MT, Skinner JS, Park EJ, Jeknic Z, Hayes PM, Thomashow MF, Chen THH. Use of a stress inducible promoter to drive ectopic $A t C B F$ expression improves potato freezing tolerance while minimizing negative effects on tuber yield. Plant Biotechnology J. 2007; 5:591-604.

30. Dubouzet JG, Sakuma Y, Ito Y, Kasuga M, Dubouzet EG, Miura S, Seki M, Shinozaki K, Yamaguchi-Shinozaki K. OsDREB genes in rice, Oryza sativa L., encode transcription activators 
that function in drought-, high-salt- and cold-responsive gene expression. Plant J. 2003; 33:751763. [PubMed: 12609047]

31. Ito Y, Katsura K, Maruyama K, Taji T, Kobayashi M, Seki M, Shinozaki K, Yamaguchi-Shinozaki K. Functional analysis of rice DREB1/CBF-type transcription factors involved in cold-responsive gene expression in transgenic rice. Plant Cell Physiol. 2006; 47:141-153. [PubMed: 16284406]

32. Qin F, Sakuma Y, Li J, Liu Q, Li Y-Q, Shinozaki K, Yamaguchi-Shinozaki K. Cloning and functional analysis of a novel $D R E B 1 / C B F$ transcription factor involved in cold-responsive gene expression in Zea mays. Plant Cell Physiol. 2004; 45:1042-1052. [PubMed: 15356330]

33. Savitch LV, Allard G, Seki M, Robert LS, Tinker NA, Huner NP, Shinozaki K, Singh J. The effect of over-expression of two Brassica CBF/DREB1-like transcription factors on photosynthetic capacity and freezing tolerance in Brassica napus. Plant Cell Physiol. 2005; 46:1525-1539. [PubMed: 16024910]

34. Welling A, Palva ET. Involvement of CBF transcription factors in winter hardiness in birch. Plant Physiol. 2008; 147:1199-1211. [PubMed: 18467468]

35. Zhang X, Fowler SG, Cheng H, Lou Y, Rhee SY, Stockinger EJ, Thomashow MF. Freezingsensitive tomato has a functional CBF cold response pathway, but a CBF regulon that differs from that of freezing-tolerant Arabidopsis. Plant J. 2004; 39:905-919. [PubMed: 15341633]

36. Gilmour SJ, Fowler SG, Thomashow MF. Arabidopsis transcriptional activators CBF1, CBF2, and CBF3 have matching functional activities. Plant Mol Biol. 2004; 54:767-781. [PubMed: 15356394]

37. Achard P, Gong F, Cheminant S, Alioua M, Hedden P, Genschik P. The cold-inducible CBF1 factor-dependent signaling pathway modulates the accumulation of the growth-repressing DELLA proteins via its effect on gibberellin metabolism. Plant Cell. 2008; 20:2117-2129. [PubMed: 18757556]

38. Chinnusamy V, Ohta M, Kanrar S, Lee B.-h, Hong X, Agarwal M, Zhu JK. ICE1, a regulator of cold induced transcriptome and freezing tolerance in Arabidopsis. Genes Dev. 2003; 17:10431054. [PubMed: 12672693]

39. Fursova OV, Pogorelko GV, Tarasov VA. Identification of ICE2, a gene involved in cold acclimation which determines freezing tolerance in Arabidopsis thaliana. Gene. 2009; 429:98103. [PubMed: 19026725]

40. Badawi M, Reddy YV, Agharbaoui Z, Tominaga Y, Danyluk J, Sarhan F, Houde M. Structure and functional analysis of wheat ICE (Inducer of CBF Expression) genes. Plant Cell Physiol. 2008; 49:1237-1249. [PubMed: 18635580]

41. Agarwal M, Hao Y, Kapoor A, Dong CH, Fujii H, Zheng X, Zhu JK. A R2R3 type MYB transcription factor is involved in the cold regulation of $C B F$ genes and in acquired freezing tolerance. J Biol Chem. 2006; 281:37636-37645. [PubMed: 17015446]

42. Miura K, Jin JB, Lee J, Yoo CY, Stirm V, Miura T, Ashworth EN, Bressan RA, Yun DJ, Hasegawa PM. SIZ1-mediated sumoylation of ICE1 controls CBF3/DREB1A expression and freezing tolerance in Arabidopsis. Plant Cell. 2007; 19:1403-1414. [PubMed: 17416732]

43. Dong CH, Agarwal M, Zhang Y, Xie Q, Zhu JK. The negative regulator of plant cold responses, HOS1, is a RING E3 ligase that mediates the ubiquiti-nation and degradation of ICE1. Proc Natl Acad Sci USA. 2006; 103:8281-8286. [PubMed: 16702557]

44. Kanaoka MM, Pillitteri LJ, Fujii H, Yoshida Y, Bogenschutz NL, Takabayashi J, Zhu JK, Torii KU. SCREAM/ICE1 and SCREAM2 specify three cell-state transitional steps leading to Arabidopsis stomatal differentiation. Plant Cell. 2008; 20:1775-1785. [PubMed: 18641265]

45. Doherty CJ, Van Buskirk HA, Myers SJ, Thomashow MF. Roles for Arabidopsis CAMTA transcription factors in cold-regulated gene expression and freezing tolerance. Plant Cell. 2009; 21:972-984. [PubMed: 19270186]

46. Novillo F, Alonso JM, Ecker JR, Salinas J. CBF2/DREB1C is a negative regulator of CBF1/ DREB1B and CBF3/DREB1A expression and plays a central role in stress tolerance in Arabidopsis. Proc Natl Acad Sci USA. 2004; 101:3985-3990. [PubMed: 15004278]

47. Novillo F, Medina J, Salinas J. Arabidopsis CBF1 andCBF3havea different function than CBF2 in cold acclimation and define different gene classes in the CBF regulon. Proc Natl Acad Sci USA. 2007; 104:21002-21007. [PubMed: 18093929] 
48. Vogel JT, et al. Roles of the CBF2 and ZAT12 transcription factors in configuring the low temperature transcriptome of Arabidopsis. Plant J. 2005; 41:195-211. [PubMed: 15634197]

49. Lee H, Guo Y, Ohta M, Xiong L, Stevenson B, Zhu JK. LOS2, a genetic locus required for cold responsive transcription encodes a bi-functional enolase. EMBO J. 2002b; 21:2692-2702. [PubMed: 12032082]

50. Xiong L, Lee H, Ishitani M, Tanaka Y, Stevenson B, Koiwa H, Bressan RA, Hasegawa PM, Zhu JK. Repression of stress-responsive genes by FIERY2, a novel transcriptional regulator in Arabidopsis. Proc Natl Acad Sci USA. 2002; 99:10899-10904. [PubMed: 12149453]

51. Fowler SG, Cook D, Thomashow MF. Low temperature induction of Arabidopsis CBF1, 2 and 3 is gated by the circadian clock. Plant Physiol. 2005; 137:961-968. [PubMed: 15728337]

52. Xin Z, Mandaokar A, Chen J, Last RL, Browse J. Arabidopsis ESK1 encodes a novel regulator of freezing tolerance. Plant J. 2007; 21:786-799. [PubMed: 17316173]

53. Zhu J, Shi H, Lee BH, Damsz B, Cheng S, Stirm V, Zhu JK, Hasegawa PM, Bressan RA. An Arabidopsis homeodomain transcription factor gene, $\mathrm{HOS} 9$, mediates cold tolerance through a CBF-independent pathway. Proc Natl Acad Sci USA. 2004; 101:9873-9878. [PubMed: 15205481]

54. Zhu J, Verslues PE, Zheng X, Lee BH, Zhan X, Manabe Y, Sokolchik I, Zhu Y, Dong CH, Zhu JK, Hasegawa PM, Bressan RA. HOS10 encodes an R2R3-type MYB transcription factor essential for cold acclimation in plants. Proc Natl Acad Sci USA. 2005; 102:9966-9971. [PubMed: 15994234]

55. Kim JC, Lee SH, Cheong YH, Yoo CM, Lee SI, Chun HJ, Yun DJ, Hong JC, Lee SY, Lim CO, Cho MJ. A novel cold-inducible zinc finger protein from soybean, SCOF-1, enhances cold tolerance in transgenic plants. Plant J. 2001; 25:247-259. [PubMed: 11208017]

56. Vannini C, Locatelli F, Bracale M, Magnani E, Marsoni M, Osnato M, Mattana M, Baldoni E, Coraggio I. Overexpression of the rice Osmyb4 gene increases chilling and freezing tolerance of Arabidopsis thaliana plants. Plant J. 2004; 37:115-127. [PubMed: 14675437]

57. Dai X, Xu Y, Ma Q, Xu W, Wang T, Xue Y, Chong K. Overexpression of a R1R2R3 MYB gene, $O s M Y B 3 R-2$, increases tolerance to freezing, drought, and salt stress in transgenic Arabidopsis. Plant Physiol. 2007; 143:1739-1751. [PubMed: 17293435]

58. Yi SY, Kim JH, Joung YH, Lee S, Kim WT, Yu SH, Choi D. The pepper transcription factor CaPF1 confers pathogen and freezing tolerance in Arabidopsis. Plant Physiol. 2004; 136:28622874. [PubMed: 15347795]

59. Xu ZS, Xia LQ, Chen M, Cheng XG, Zhang RY, Li LC, Zhao YX, Lu Y, Ni ZY, Liu L, Qiu ZG, Ma YZ. Isolation and molecular characterization of the Triticum aestivum L. ethylene-responsive factor 1 (TaERF1) that increases multiple stress tolerance. Plant Mol Biol. 2007; 65:719-732. [PubMed: 17874224]

60. Liu N, Zhong NQ, Wang GL, Li LJ, Liu XL, He YK, Xia GX. Cloning and functional characterization of PpDBF1 gene encoding a DRE-binding transcription factor from Physcomitrella patens. Planta. 2007; 226:827-838. [PubMed: 17541631]

61. Chen M, Xu Z, Xia L, Li L, Cheng X, Dong J, Wang Q, Ma Y. Cold-induced modulation and functional analyses of the DRE-binding transcription factor gene, GmDREB3, in soybean (Glycine $\max$ L.). J Exp Bot. 2009; 60:121-135. [PubMed: 18988621]

62. Kobayashi F, Maeta E, Terashima A, Kawaura K, Ogihara Y, Takumi S. Development of abiotic stress tolerance via bZIP-type transcription factor LIP19 in common wheat. J Exp Bot. 2008; 59:891-905. [PubMed: 18326864]

63. Hu H, You J, Fang Y, Zhu X, Qi Z, Xiong L. Characterization of transcription factor gene SNAC2 conferring cold and salt tolerance in rice. Plant Mol Biol. 2008; 67:169-181. [PubMed: 18273684]

64. Mastrangelo AM, Belloni S, Barilli S, Ruperti B, Fonzo ND, Stanca AM, Cattivelli L. Low temperature promotes intron retention in two $e$-cor genes of durum wheat. Planta. 2005; 221:705715. [PubMed: 15666155]

65. Nakayama K, Okawa K, Kakizaki T, Honma T, Itoh H, Inaba T. Arabidopsis Cor15am is a chloroplast stromal protein that has cryoprotective activity and forms oligomers. Plant Physiol. 2007; 144:513-523. [PubMed: 17384167] 
66. Lee BH, Kapoor A, Zhu J, Zhu JK. STABILIZED1, a stress-upregulated nuclear protein, is required for pre-mRNA splicing, mRNA turnover, and stress tolerance in Arabidopsis. Plant Cell. 2006; 18:1736-1749. [PubMed: 16751345]

67. Palusa SG, Ali GS, Reddy ASN. Alternative splicing of pre-mRNAs of Arabidopsis serine/ arginine-rich proteins: regulation by hormones and stresses. Plant J. 2007; 49:1091-1107. [PubMed: 17319848]

68. Tanabe N, Yoshimura K, Kimura A, Yabuta Y, Shigeoka S. Differential expression of alternatively spliced mRNAs of Arabidopsis SR protein homologs, atSR30 and atSR45a, in response to environmental stress. Plant Cell Physiol. 2007; 48:1036-1049. [PubMed: 17556373]

69. Sunkar R, Chinnusamy V, Zhu J, Zhu JK. Small RNAs as big players in plant abiotic stress responses and nutrient deprivation. Trends Plant Sci. 2007; 12:301-309. [PubMed: 17573231]

70. Sunkar R, Kapoor A, Zhu JK. Posttranscriptional induction of two Cu/Zn superoxide dismutase genes in Arabidopsis is mediated by downregulation of miR398 and important for oxidative stress tolerance. Plant Cell. 2006; 18:2051-2065. [PubMed: 16861386]

71. Borsani O, Zhu J, Verslues PE, Sunkar R, Zhu JK. Endogenous siRNAs derived from a pair of natural cisantisense transcripts regulate salt tolerance in Arabidopsis. Cell. 2005; 123:1279-1291. [PubMed: 16377568] 
Table 3.1

Abiotic stress tolerance of transgenic plants overexpressing CBFs

\begin{tabular}{|c|c|c|c|}
\hline Gene & Transgenic plant & Stress tolerance of transgenic plants & References \\
\hline AtCBF $1 / 2 / 3$ & Brassica napus & Constitutive overexpression enhanced both basal and acquired freezing tolerance & (22) \\
\hline$A t C B F 1$ & Tomato & $\begin{array}{l}\text { Constitutive overexpression enhanced oxidative stress tolerance under chilling } \\
\text { stress; enhanced tolerance to water-deficit stress }\end{array}$ & $(23,24)$ \\
\hline$A t D R E B 1 A / C B F 3$ & Tobacco & $\begin{array}{l}\text { Transgenic plants expressing } R D 29 A:: D R E B 1 A \text { exhibited enhanced chilling and } \\
\text { drought tolerance }\end{array}$ & $(25)$ \\
\hline$A t D R E B 1 A / C B F 3$ & Wheat & $\begin{array}{l}\text { Transgenic plants expressing } R D 29 A \text { promoter::AtDREB } 1 A \text { gene showed } \\
\text { delayed water stress symptoms }\end{array}$ & $(26)$ \\
\hline$A t C B F 3$ & Rice & $\begin{array}{l}\text { Constitutive overexpression resulted in enhanced tolerance to drought and high } \\
\text { salinity and a marginal increase in chilling tolerance }\end{array}$ & $(27)$ \\
\hline$A t D R E B 1 A / C B F 3$ & Maize & $R D 29 A:: C B F 3$ transgenic plants are more tolerant to cold, drought, and salinity & (28) \\
\hline$A t C B F 1$ & Potato & $\begin{array}{l}\text { Constitutive or stress-inducible expression of } C B F 1 \text { or } C B F 3 \text { but not } C B F 2 \\
\text { conferred improved freezing tolerance to frost-sensitive Solanum tuberosum }\end{array}$ & (29) \\
\hline OsDREB1 & Arabidopsis & $\begin{array}{l}\text { Overexpression in Arabidopsis induced target COR genes and conferred } \\
\text { enhanced tolerance to freezing and drought stresses }\end{array}$ & (30) \\
\hline$O s D R E B 1 A / B$ & Rice & $\begin{array}{l}\text { Constitutive expression conferred improved tolerance to cold, drought, and } \\
\text { salinity }\end{array}$ & (31) \\
\hline ZmDREBI & Arabidopsis & $\begin{array}{l}\text { Overexpression in Arabidopsis induced } C O R \text { genes and conferred tolerance to } \\
\text { freezing and drought }\end{array}$ & (32) \\
\hline $\begin{array}{l}B n C B F 5 \text { and } B n C B F \\
17\end{array}$ & B. napus & $\begin{array}{l}\text { Overexpression led to increased constitutive freezing tolerance, increased } \\
\text { photochemical efficiency and photosynthetic capacity }\end{array}$ & (33) \\
\hline
\end{tabular}

